\title{
Analysis theorem of unique common fixed point for four maps based on partial -b- metric spaces
}

\author{
Ban Mohammad Hasan, Hayder Abdulameer Abbas
}

Middle Technical University, Technical Instructors Training Institute, Baghdad, Iraq

\begin{tabular}{|c|c|}
\hline Article Info & ABSTRACT \\
\hline Article history: & A In this paper, An important definitions are to be used to prove the existence \\
\hline Received Oct 6, 2018 & $\begin{array}{l}\text { of a common tixed point theorem for four mappings incomplete, partial }-\mathrm{b}- \\
\text { metric spaces, as well as prove a unique common fixed point by assuming }\end{array}$ \\
\hline Revised Nov 21, 2018 & another point and getting that, these points are finally equal. We presented an \\
\hline Accepted Dec 3, 2018 & example thus enhancing us the outcome. \\
\hline
\end{tabular}

\section{Keywords: \\ Curtailment \\ Partial b-metric space \\ Partial metric space \\ Weakly compatible mapping} \begin{abstract}
metric spaces, as well as prove a unique common fixed point by assuming example thus enhancing us the outcome.
\end{abstract}

\section{Corresponding Author:}

Ban Mohammad Hasan,

Technical Instructors Training Institute,

Middle Technical University, Baghdad, Iraq.

Email: haeder_abid@yahoo.com

\section{INTRODUCTION}

In 1989, Bakhtin [1] approved the idea respecting a quasi-metric space as a generalized concept about metric spaces. In 1993, Czerwik [2, 3] expanded abundant upshots concerning for b- metric spaces. In 1994, Matthews [4] found the connotation concerning partial metric space at the self - distance in connection with any point about space might not equal zero. In 1996, O'Neill assured that a connotation for partial metric space through granting negative distances. In 2013, Shukla [5] assured together the connotation about b-metric \& partial metric spaces via send in the partial b-metric spaces. For example, researchers explored the concept \& its generalizations in several kinds of metric spaces [6-10].

Within this research, we proved a common fixed point theorem for four maps in partial $b$ - metric space and in this paper we generalize both the concepts of b-metric and partial metric spaces by introducing the partial $b$-metric space. An analog of the common fixed point theorem for four maps in partial $b-$ metric spaces is proved. Some examples are included which illustrate the results obtained in new space. First, we recall some definitions from b-metric and partial metric spaces.

Definition 1.1. [11-13] Let $X$ be a nonempty set and let $s \geq 1$ be a given real number. A function $d: X \times X \rightarrow[0, \infty)$ is called $a \mathrm{~b}$-metric if for all $\mathrm{x}, \mathrm{y}, \mathrm{z} \in \mathrm{X}$ the following conditions are satisfied:

(i) $d(x, y)=0$ if and only if $x=y$;

(ii) $\mathrm{d}(\mathrm{x}, \mathrm{y})=\mathrm{d}(\mathrm{y}, \mathrm{x})$;

(iii) $d(x, y) \leq s[d(x ; z)+d(z ; y)]$ :

The pair $(X, d)$ is called a b-metric space. The number $s \geq 1$ is called the coefficient of $(X, d)$

Definition 1.2. [4] Let $X$ be a nonempty set. A function $p: X \times X \rightarrow[0, \infty)$ is called a partial metric if for all $x$, $\mathrm{y}, \mathrm{z} \in \mathrm{X}$ the following conditions are satisfied: 
(i) $x=y$ if and only if $p(x, x)=p(x, y)=p(y, y)$;

(ii) $\mathrm{p}(\mathrm{x}, \mathrm{x}) \leq \mathrm{p}(\mathrm{x}, \mathrm{y})$;

(iii) $\mathrm{p}(\mathrm{x}, \mathrm{y})=\mathrm{p}(\mathrm{y}, \mathrm{x})$;

(iv) $\mathrm{p}(\mathrm{x}, \mathrm{y}) \leq \mathrm{p}(\mathrm{x}, \mathrm{z})+\mathrm{p}(\mathrm{z}, \mathrm{y})-\mathrm{p}(\mathrm{z} ; \mathrm{z})$ :

The pair $(X ; d)$ is called a partial metric space.

Remark 1.3 Apparent the partial metric space not necessity be a metric spaces, $\because$ in a $b$ - metric space whether $\mathrm{v}=\mathrm{w}, \Rightarrow \mathrm{d}(\mathrm{v}, \mathrm{v})=\mathrm{d}(\mathrm{v}, \mathrm{w})=\mathrm{d}(\mathrm{w}, \mathrm{w})=0$. in a partial metric space if $\mathrm{v}=\mathrm{w}$

$\Rightarrow p(v, v)=p(v, w)=p(w, w)$ not necessary $b=0$. Thence the partial metric space not necessary be a $b-$ metric space.

So the else direction, Shukla [5] pressed the connotation of a partial b-metric space as pursue:

Definition 1.4. [5] If $\mathrm{V}$ be $\neq \varnothing$ set $\& \mathrm{~S} \geq 1$ be a given $\mathbb{R}$. function

$P_{\mathrm{b}}: V \times V \rightarrow[0, \infty)$ is expressing a partial $\mathrm{b}-$ metric if $\forall \mathrm{v}, \mathrm{w}, \mathrm{z} \in \mathrm{V}$ the following conditions are convinced: i: $\mathrm{v}=\mathrm{w} \Leftrightarrow \mathrm{P}_{\mathrm{b}}(\mathrm{v}, \mathrm{v})=\mathrm{P}_{\mathrm{b}}(\mathrm{v}, \mathrm{w})=\mathrm{P}_{\mathrm{b}}(\mathrm{w}, \mathrm{w})$;

ii: $P_{b}(v, v) \leq P_{b}(v, w)$;

iii: $\mathrm{P}_{\mathrm{b}}(\mathrm{v}, \mathrm{w})=\mathrm{P}_{\mathrm{b}}(\mathrm{w}, \mathrm{v})$;

iv: $\mathrm{P}_{\mathrm{b}}(\mathrm{v}, \mathrm{w}) \leq \dot{\mathrm{S}}\left[\mathrm{P}_{\mathrm{b}}(\mathrm{v}, \mathrm{z})+\mathrm{P}_{\mathrm{b}}(\mathrm{z}, \mathrm{w})\right]-\mathrm{P}_{\mathrm{b}}(\mathrm{z} ; \mathrm{z})$ :

The $\left(\mathrm{V} ; \mathrm{P}_{\mathrm{b}}\right)$ is expressing a partial $\mathrm{b}$-metric space. The amount $\mathrm{s} \geq 1$ the parameter is called $\left(\mathrm{V}, \mathrm{P}_{\mathrm{b}}\right)$.

Remark 1.5. The kind of partial b-metric space $\left(\mathrm{V}, \mathrm{P}_{\mathrm{b}}\right)$ is the most effective way the kind of partial metric space $\because$ a partial metric space is a condition shape from a partial b-metric space. $\left(V, P_{b}\right)$ while $s=1$. Likewise, the kind of partial b-metric space $\left(\mathrm{V}, \mathrm{P}_{\mathrm{b}}\right)$ is effective way bigger than the kind from $\mathrm{b}$-metric space, $\because$ a b-metric space is a private condition from a partial $\mathrm{b}$-metric space $\left(\mathrm{V}, \mathrm{P}_{\mathrm{b}}\right)$ while the same - area $\mathrm{p}(\mathrm{v} ; \mathrm{v})=0$.

The next exa. articulate this one a partial b-metric on $\mathrm{V}$ requirement not be a partial metric, neither a b-metric on V, look as well [14], [5].

Example 1.6. [5] Allowed $V=[0,1)$. Realize a function $P_{\mathrm{b}}: V \times V \rightarrow[0, \infty)$ S.T.

$\mathrm{P}_{\mathrm{b}}(\mathrm{v} ; \mathrm{w})=[\max .\{\mathrm{v}, \mathrm{w}\}]^{2}+|\mathrm{v}-\mathrm{w}|^{2}, \forall \mathrm{v}, \mathrm{w} \in \mathrm{V}$ therefor $\left(\mathrm{V}, \mathrm{P}_{\mathrm{b}}\right)$ is a partial b-metric metric \& also not a partial metric to $\mathrm{V}$.

Definition 1.7. [14] Any partial b-metric $\mathrm{P}_{\mathrm{b}}$ is known a b- metric $d_{p_{\mathrm{b}}}$ whosesoever $d_{p_{\mathrm{b}}}(\mathrm{v}, \mathrm{w})=2 \mathrm{P}_{\mathrm{b}}(\mathrm{v} ; \mathrm{w})-\mathrm{P}_{\mathrm{b}}(\mathrm{v}, \mathrm{v})-\mathrm{P}_{\mathrm{b}}(\mathrm{w}, \mathrm{w}), \forall \mathrm{v}, \mathrm{w} \in \mathrm{V}$.

Definition 1.8. [14] A sequence $\left\{\mathrm{v}_{\mathrm{n}}\right\}$ in a partial b-metric space $\left(\mathrm{V}, \mathrm{P}_{\mathrm{b}}\right)$ is called: 1- $\mathrm{P}_{\mathrm{b}}$-convergent for $\mathrm{v} \in \mathrm{V}$ if $\lim _{n \rightarrow \infty} \mathrm{P}_{\mathrm{b}}\left(\mathrm{V}, v_{n}\right)=\mathrm{P}_{\mathrm{b}}(\mathrm{v}, \mathrm{v})$

2- $\mathrm{P}_{\mathrm{b}}$ - Cauchy sequence if $\lim _{n, m \rightarrow \infty} \mathrm{P}_{\mathrm{b}}\left(v_{n}, \mathrm{v}_{m}\right)$ subsist \&is finite;

3- partial b-metric space $\left(V, P_{b}\right)$ became $P_{b}$-complete whether $\forall P_{b}-$ Cauchy sequence $\left\{v_{n}\right\}$ in $V$ is $P_{b}$ converges for $\mathrm{v} \in \mathrm{V}, \mathrm{S}$.T.

$$
\lim _{n, m \rightarrow \infty} \mathrm{P}_{\mathrm{b}}\left(v_{n}, \mathrm{v}_{m}\right)=\lim _{n \rightarrow \infty} \mathrm{P}_{\mathrm{b}}\left(v_{n}, \mathrm{v}\right)=\mathrm{P}_{\mathrm{b}}(\mathrm{v}, \mathrm{v})
$$

Lemma 1.9. [14] A sequence $\left\{x_{n}\right\}$ is a $P_{b}$-Cauchy sequence in a partial b-metric space $\left(X, P_{b}\right)$ if and only if it is a b-Cauchy sequence in the b-metric space $\left(\mathrm{X}, d_{P_{b}}\right)$.

Lemma 1.10. [14] A partial b-metric space $\left(X, P_{b}\right)$ is $P_{b}$-complete if and only if the b-metric space $\left(X, d_{P_{b}}\right)$ is b-complete. Moreover, $\lim _{n, m \rightarrow \infty} d_{P_{b}}\left(x_{n}, \mathrm{x}_{m}\right)=0$ if and only if

$$
\lim _{n, m \rightarrow \infty} \mathrm{P}_{b}\left(x_{m}, \mathrm{x}\right)=\lim _{n \rightarrow \infty} \mathrm{P}_{b}\left(x_{n}, \mathrm{x}\right)=\mathrm{P}_{b}(\mathrm{x}, \mathrm{x})
$$

Definition 1.11 [15]: A \& S two self-maps from a metric space (V,d) are designation weakly compatible if, at coincidence points those commute. Which, in case $\mathrm{Av}=\mathrm{Sv} \Rightarrow \mathrm{ASv}=\mathrm{SAv}$ for $\mathrm{v}$ in $\mathrm{V}$.

Presently we demonstrate our essential outcome. 


\section{MAIN RESULTS}

Theorem: 2.1: aLet $\left(w, p_{\mathrm{b}}\right)$ be a partial $\mathrm{b}-$ metric space for the coefficient $\dot{S} \geq 1$, consign $A, \mathrm{~B}, C$ $, \mathrm{D}: \mathrm{V} \rightarrow \mathrm{V}$ be mappings appropriate the next

(2.1.1) S. $p_{\mathrm{b}}\left(\mathrm{A}_{V}, \mathrm{~B}_{w}\right) \leq \max .\left\{\begin{array}{c}p_{\mathrm{b}}\left(\mathrm{C}_{V}, \mathrm{Ð}_{W}\right), P_{\mathrm{b}}\left(\mathrm{C}_{V}, \mathrm{~A}_{V}\right), P_{\mathrm{b}}\left(\oplus_{w}, \mathrm{~B}_{w)}\right) \\ \frac{1}{2 \tilde{S}}\left[P_{\mathrm{b}}\left(\mathrm{C}_{w}, \mathrm{~B}_{w}\right)+P_{\mathrm{b}}\left(\bigoplus_{w}, \mathrm{~A}_{V}\right)\right]\end{array}\right\}$

Where $\mathrm{K} \in\left[0, \frac{1}{\tilde{\mathrm{S}}}\right), \forall v, w \in V$

(2.1.2) $\mathrm{A}(V) \subseteq \mathrm{Ð}(V), \mathrm{B}(V) \subseteq C(V)$

(2.1.3) with regard to $\mathrm{C}(\mathrm{V})$ or $Đ(V)$ is complete subspace of $\mathrm{V}$.

(2.1.4) the (A; C) \& (B, Đ) are weakly compatible.

So $A, B, C \zeta \&$ include unique common fixed point in $\mathrm{V}$

Proof : Select $v_{0}, w_{0} \in V$. From (2.1.2) $\exists$, sequences $\left\{v_{n}\right\} \&\left\{w_{n}\right\}$ in V s.t.

$\mathrm{A} v_{2 n}=Đ v_{2 n+1}=w_{2 n}$

$\mathrm{B} v_{2 n+1}=C_{1} v_{2 n+2}=w_{2 n+1} \forall \mathrm{n}=0,1,2,3$,

Status: (i):-Assume $w_{2 n}=w_{2 n+1}$ for some $\mathrm{n}$.

Clam: $\quad w_{2 n+1}=w_{2 n+2}$

Supp. $w_{2 n+1} \neq w_{2 n+2}$

From (2.1.1), then

$$
\begin{aligned}
& \text { Ś. } p_{\mathrm{b}}\left(w_{2 n+1}, w_{2 n+2}\right)=\text { Ś. } p_{\mathrm{b}}\left(\mathrm{A} v_{2 n+2}, \mathrm{~B} v_{2 n+1}\right) \\
& \leq \mathrm{k} \max .\left\{\begin{array}{c}
P_{\mathrm{b}}\left(\mathrm{C} v_{2 n+2}, \mathrm{Ð} v_{2 n+1}\right), P_{\mathrm{b}}\left(\mathrm{C} v_{2 n+2}, \mathrm{~A} v_{2 n+1}\right), P_{\mathrm{b}}\left(\mathrm{\oplus} v_{2 n+1}, \mathrm{~B} v_{2 n+1}\right), \\
\frac{1}{2 \tilde{S}}\left[P_{\mathrm{b}}\left(\mathrm{C} v_{2 n+1}, \mathrm{~B} v_{2 n+1}\right)+P_{\mathrm{b}}\left(\mathrm{\oplus} v_{2 n+1}, \mathrm{~A} v_{2 n+1}\right)\right]
\end{array}\right\} \\
& =\mathrm{k} \max .\left\{\begin{array}{c}
p_{\mathrm{b}}\left(w_{2 n+1}, w_{2 n}\right), P_{\mathrm{b}}\left(w_{2 n+1}, w_{2 n+2}\right), P_{\mathrm{b}}\left(w_{2 n}, w_{2 n+1}\right), \\
\frac{1}{2 \hat{S}}\left[P_{\mathbf{b}}\left(w_{2 n+1}, w_{2 n+1}\right)+P_{\mathrm{b}}\left(w_{2 n+1}, w_{2 n+2}\right)\right]
\end{array}\right\} \\
& =\mathrm{k} \max \left\{\begin{array}{c}
p_{\mathrm{b}}\left(w_{2 n+1}, w_{2 n+1}\right), P_{\mathrm{b}}\left(w_{2 n+1}, w_{2 n+2}\right), P_{\mathrm{b}}\left(w_{2 n+1}, w_{2 n+1}\right), \\
\frac{1}{2 \tilde{S}}\left[P_{\mathrm{b}}\left(w_{2 n+1}, w_{2 n+1}\right)+P_{\mathrm{b}}\left(w_{2 n}, w_{2 n+2}\right)\right]
\end{array}\right\} \\
& =\mathrm{k} p_{\mathrm{b}}\left(w_{2 n+1}, w_{2 n+2}\right) \text {, }
\end{aligned}
$$

that is a discrepancy.

$$
\therefore w_{2 n+1}=w_{2 n+2}
$$

Stay in same direction we ability ratiocinate that

$w_{2 n}=w_{2 n+k}$

$\therefore\left\{w_{2 n}\right\}$ a Cauchy sequence in $\mathrm{V}$

Status (ii):- $\quad w_{n} \neq w_{n+1} \forall n$.

From (2.1.1), consider

$$
\begin{aligned}
& \text { S. } p_{\mathrm{b}}\left(\mathrm{A} v_{2 n}, \mathrm{~B} v_{2 n+1}\right) \leq \max \left\{\begin{array}{c}
p_{\mathrm{b}}\left(\mathrm{C} v_{2 n}, \mathrm{\bigoplus} v_{2 n+1}\right), P_{\mathrm{b}}\left(\mathrm{C} v_{2 n}, \mathrm{~A} v_{2 n}\right), P_{\mathrm{b}}\left(\mathrm{Ð} v_{2 n+1}, \mathrm{~B} v_{2 n+1}\right), \\
\frac{1}{2 \dot{S}}\left[P_{\mathrm{b}}\left(\mathrm{C} v_{2 n}, \mathrm{~B} v_{2 n+1}\right)+P_{\mathrm{b}}\left(\mathrm{Ð} v_{2 n+1}, \mathrm{~A} v_{2 n}\right)\right]
\end{array}\right\} \\
& =\mathrm{k} \max \left\{\begin{array}{c}
p_{\mathrm{b}}\left(w_{2 n-1}, w_{2 n}\right), P_{\mathrm{b}}\left(w_{2 n-1}, w_{2 n}\right), P_{\mathrm{b}}\left(w_{2 n}, w_{2 n+1}\right), \\
\frac{1}{2 \dot{S}}\left[P_{\mathrm{b}}\left(w_{2 n-1}, w_{2 n+1}\right)+P_{\mathrm{b}}\left(w_{2 n}, w_{2 n}\right)\right]
\end{array}\right\}
\end{aligned}
$$




$$
\begin{aligned}
& =\mathrm{k} \max \left\{\begin{array}{c}
p_{\mathrm{b}}\left(w_{2 n-1}, w_{2 n}\right), P_{\mathrm{b}}\left(w_{2 n}, w_{2 n}\right), P_{\mathrm{b}}\left(w_{2 n}, w_{2 n+1}\right), \\
\frac{1}{2 \dot{S}}\left[\hat{S}\left[P_{\mathrm{b}}\left(w_{2 n-1}, w_{2 n}\right)+P_{\mathrm{b}}\left(w_{2 n}, w_{2 n+1}\right)\right]\right]
\end{array}\right\} \\
& =\mathrm{k} \max \left\{p_{\mathrm{b}}\left(w_{2 n-1}, w_{2 n}\right), p_{\mathrm{b}}\left(w_{2 n}, w_{2 n+1}\right)\right\} \\
& \text { if } p_{\mathrm{b}}\left(w_{2 n}, w_{2 n+1}\right) \text { is maximum, then } \\
& \text { S. } p_{\mathrm{b}}\left(w_{2 n}, w_{2 n+1}\right) \leq k p_{\mathrm{b}}\left(w_{2 n}, w_{2 n+1}\right)
\end{aligned}
$$

which implies

$$
p_{\mathrm{b}}\left(w_{2 n}, w_{2 n+1}\right) \leq \frac{K}{\dot{S}} p_{\mathrm{b}}\left(w_{2 n}, w_{2 n+1}\right)<p_{\mathrm{b}}\left(w_{2 n}, w_{2 n+1}\right)
$$

which is a contradiction.

Hence $p_{\mathrm{b}}\left(w_{2 n-1}, w_{2 n}\right)$ is maximum. So that

$$
\text { S.. } p_{\mathrm{b}}\left(w_{2 n}, w_{2 n+1}\right) \leq k p_{\mathrm{b}}\left(w_{2 n-1}, w_{2 n}\right)
$$

implies that

$$
p_{\mathrm{b}}\left(w_{2 n}, w_{2 n+1}\right) \leq \frac{K}{S} p_{\mathrm{b}}\left(w_{2 n-1}, w_{2 n}\right)
$$

Put $p_{2 n}=p_{\mathrm{b}}\left(w_{2 n}, w_{2 n+1}\right)$

Then $\left\{P_{2 n}\right\}$ is decreasing sequence of non-negative $\mathbb{R} \&$ must converges to some $\mathbb{R}$

$l \geq 0$. (say)

Assume $l>0$

Letting $n \rightarrow \infty$ in (1), we obtain

$$
l \leq \frac{k}{s} \cdot e l<l
$$

Is the antinomy.

$\Rightarrow l=0$. So

$$
\lim _{n \rightarrow \infty} p_{\mathrm{b}}\left(\mathrm{w}_{2 \mathrm{n}}, \mathrm{w}_{2 \mathrm{n}+1}\right)=0
$$

Hence for def.1.4

$$
\lim _{n \rightarrow \infty} p_{\mathrm{b}}\left(w_{2 n}, w_{2 n}\right)=0
$$

From (2) and (3) and by definition of $d_{P_{b}}$, we get

$$
\lim _{n \rightarrow \infty} d_{P_{\mathrm{b}}}\left(w_{2 n}, w_{2 n+1}\right)=0 \text {. }
$$

For $\mathrm{m}, \mathrm{n} \in N$ with $\mathrm{m}>\mathrm{n}$, we have 


$$
\begin{aligned}
& p_{\mathrm{b}}\left(w_{2 n}, w_{2 m}\right) \leq \dot{S}\left[p_{\mathrm{b}}\left(w_{2 n}, w_{2 n+1}\right)+p_{\mathrm{b}}\left(w_{2 n+1}, w_{2 m}\right)\right]-p_{\mathrm{b}}\left(w_{2 n+1}, w_{2 n+1}\right) \\
& \leq \dot{S} \cdot p_{\mathrm{b}}\left(w_{2 n}, w_{2 n+1}\right)+\dot{S}^{2} p_{\mathrm{b}}\left(w_{2 n+1}, w_{2 n+2}\right)+\cdots+\dot{S}^{2 m-2 n} p_{\mathrm{b}}\left(w_{2 m-1}, w_{2 m}\right) \\
& \leq \dot{S} \cdot \frac{K^{2 n+1}}{\dot{S}^{2 n+1}} p_{\mathrm{b}}\left(w_{0}, w_{1}\right)+\dot{S}^{2} \frac{K^{2 n+2}}{\dot{S}^{2 n+2}} p_{\mathrm{b}}\left(w_{0}, w_{1}\right)+\cdots+\dot{S}^{2 m-2 n} \frac{K^{2 m}}{\dot{S}^{2 m}} p_{\mathrm{b}}\left(w_{0}, w_{1}\right) \\
& =\frac{K^{2 n}}{\dot{S}^{2 n}}\left[k+k^{2}+k^{3}+\cdots+k^{2 m-2 n}\right] \cdot p_{\mathrm{b}}\left(w_{0}, w_{1}\right) \\
& \quad k \in\left[0, \frac{1}{s}\right) \& \dot{\mathrm{S}} \geq 1, \text { it follows from the above then } \\
& \text { As } \quad \lim _{n, m \rightarrow \infty} p_{\mathrm{b}}\left(w_{2 n}, w_{2 m}\right)=0
\end{aligned}
$$

Then $\left\{w_{2 n}\right\}$ is a Cauchy sequence in $\mathrm{V}$

Same that we competence likewise evince that $\left\{w_{2 n+1}\right\}$ is a Cauchy sequence in V.

Subsequently $\left\{w_{2 n}\right\}$ is a Cauchy sequence in $\mathrm{V}$.

According to Lemma (1.9), we name it $\left\{w_{2 n}\right\}$ is a Cauchy sequence in $\left(\mathrm{v}, d_{P_{\mathrm{b}}}\right)$.

Suppose $\mathrm{C}(\mathrm{v})$ is a complete subspace of $\mathrm{V}$.

$\because\left\{w_{2 n+1}\right\}$ is a Cauchy sequence in complete b-metric space $\left(\mathrm{C}(\mathrm{v}), d_{P_{\mathrm{b}}}\right)$.

This is a follow-up $\left\{w_{2 n+1}\right\}$ converges to $w$ in $Đ(V)$. So

$\lim$

$$
\lim _{n, \rightarrow \infty} d_{P_{\mathrm{b}}}\left(w_{2 n+1}, w\right)=0
$$

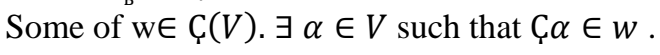

$\because\left\{w_{2 n+1}\right\}$ is Cauchy sequence $\& w_{2 n+1} \rightarrow w$.

It follows that $w_{2 n} \rightarrow w$

According to Lemma (1.10) \& (4), we possess that

$$
\begin{aligned}
& \left.p_{\mathrm{b}}(w, w)=\lim _{n \rightarrow \infty} p_{\mathrm{b}}\left(w_{2 n}, \mathrm{w}\right)=\lim _{n \rightarrow \infty} p_{\mathrm{b}} w_{2 n+1}, \mathrm{w}\right)=0 \\
& \lim _{n \rightarrow \rightarrow \infty} p_{\mathrm{b}}\left(\mathrm{A} \alpha, w_{2 n}\right)= \\
& p_{\mathrm{b}}(\mathrm{A} \alpha, w)
\end{aligned}
$$

$\because$ that def. of $d_{P_{b}}$,

$d_{P_{\mathrm{b}}}\left(\AA \alpha, w_{2 n}\right)=2 p_{\mathrm{b}}\left(\AA \alpha, w_{2 n}\right)-p_{\mathrm{b}}(\AA \alpha, \AA \alpha)-p_{\mathrm{b}}\left(w_{2 n}, w_{2 n}\right)$

According to def. of $d_{P_{b}}$ (4) \& (5), we possess that

$$
d_{P_{\mathrm{b}}}(\mathrm{A} \alpha, w)=\lim _{n \rightarrow \infty} 2 \cdot p_{b}\left(\AA \alpha, w_{2 n}\right)
$$

implies that 
$\lim$

${ }_{n \rightarrow \infty} p_{\mathrm{b}}\left(\AA \alpha, w_{2 n}\right)=p_{\mathrm{b}}(\AA \alpha, w)$

From, def. (1.4) we have

$$
\begin{aligned}
& \quad p_{\mathrm{b}}(\mathrm{A} \alpha, w) \leq \dot{S}\left[p_{\mathrm{b}}\left(\mathrm{A} \alpha, w_{2 n+1}\right)+p_{\mathrm{b}}\left(w_{2 n+1}, w\right)\right]-p_{\mathrm{b}}\left(w_{2 n+1}, w_{2 n+1}\right) \\
& =\dot{S}\left[p_{\mathrm{b}}\left(\mathrm{A} \alpha, w_{2 n+1}\right)+p_{\mathrm{b}}\left(w_{2 n+1}, w\right)\right] \\
& \text { Allowing } n \rightarrow \infty, \\
& \therefore p_{\mathrm{b}}(\mathrm{A} \alpha, w) \leq \dot{S} \cdot \lim _{n \rightarrow \infty} p_{\mathrm{b}}\left(\mathrm{A} \alpha, w_{2 n+1}\right) \\
& =\lim _{n \rightarrow \infty} \dot{S} \cdot p_{\mathrm{b}}\left(\mathrm{A} \alpha, \mathrm{B} v_{2 n+1}\right) \leq \lim _{n \rightarrow \infty} k \cdot \max \left\{\begin{array}{c}
p_{\mathrm{b}}\left(\mathrm{C} \alpha, \boxminus v_{2 n+1}\right), P_{\mathrm{b}}(\mathrm{C} \alpha, \mathrm{A} \alpha), P_{\mathrm{b}}\left(\mathrm{Ð} v_{2 n+1}, \mathrm{~B} v_{2 n+1}\right), \\
\frac{1}{2 \dot{S}}\left[P_{\mathrm{b}}\left(\mathrm{C} \alpha, \mathrm{B} v_{2 n+1}\right)+P_{\mathrm{b}}\left(\mathrm{Ð} v_{2 n+1}, \mathrm{~A} \alpha\right)\right]
\end{array}\right\} \\
& =\lim _{n \rightarrow \infty} \mathrm{k} \cdot \max \left\{\begin{array}{c}
p_{\mathrm{b}}\left(w, w_{2 n}\right), P_{\mathrm{b}}(w, \mathrm{~A} \alpha), P_{\mathrm{b}}\left(w_{2 n}, w_{2 n+1}\right), \\
\frac{1}{2 \dot{S}}\left[P_{\mathrm{b}}\left(w, w_{2 n+1}\right)+P_{\mathrm{b}}\left(w_{2 n}, \mathrm{~A} \alpha\right)\right]
\end{array}\right\} \\
& =\mathrm{k} \cdot p_{\mathrm{b}}(\mathrm{A} \alpha, w)
\end{aligned}
$$

It is clear that $\AA \alpha=w=C_{\Upsilon} \alpha$.

$\because$ the pair $(\mathrm{A}, \mathrm{C})$ is a weakly compatible pair, we hold

$\mathrm{Aw}=\mathrm{C}$ w

Here we demonstrate that $. \mathrm{Aw}=\mathrm{w}$. Consider

$p_{\mathrm{b}}(\mathrm{A} w, w) \leq \dot{S}\left[p_{\mathrm{b}}\left(\mathrm{A} w, w_{2 n+1}\right)+p_{\mathrm{b}}\left(w_{2 n+1}, w\right)\right]-p_{\mathrm{b}}\left(w_{2 n+1}, w_{2 n+1}\right)$

$\leq \dot{S}\left[p_{\mathrm{b}}\left(\mathrm{A} w, w_{2 n+1}\right)+p_{\mathrm{b}}\left(w_{2 n+1}, w\right)\right]$

Allowing $n \rightarrow \infty$,

$\therefore p_{\mathrm{b}}(\mathrm{A} w, w) \leq \mathrm{S}^{\lim _{n \rightarrow \infty}} p_{\mathrm{b}}\left(\mathrm{A} w, \mathrm{~B} x_{2 n+1}\right)$

$\lim _{n \rightarrow \infty} k \cdot \max \left\{\begin{array}{c}p_{\mathrm{b}}\left(\mathrm{C} w, \mathrm{Ð} v_{2 n+1}\right), P_{\mathrm{b}}(w, \mathrm{~A} w), P_{\mathrm{b}}\left(\mathrm{Ð} v_{2 n+1}, \mathrm{~B} v_{2 n+1}\right), \\ \frac{1}{2 \dot{S}}\left[P_{\mathrm{b}}\left(\mathrm{C} w, \mathrm{~B} v_{2 n+1}\right)+P_{\mathrm{b}}\left(\mathrm{Ð} v_{2 n+1}, \mathrm{~A} w\right)\right]\end{array}\right\} \mathrm{q}$

$=\lim _{n \rightarrow \infty} \mathrm{k} \cdot \max \left\{\begin{array}{c}p_{\mathrm{b}}\left(\mathrm{A} w, w_{2 n}\right), P_{\mathrm{b}}(\mathrm{A} w, \mathrm{~A} w), P_{\mathrm{b}}\left(w_{2 n}, w_{2 n+1}\right), \\ \frac{1}{2 \tilde{S}}\left[P_{\mathrm{b}}\left(\mathrm{A} w, w_{2 n+1}\right)+P_{\mathrm{b}}\left(\mathrm{A} w, w_{2 n}\right)\right]\end{array}\right\}$

$=k p_{\mathrm{b}}(\mathrm{A} w, w)$.

It is clear that $\mathrm{Aw}=\mathrm{W}$.

$\therefore \quad \mathrm{w}$ is common fixed point of $\mathrm{A} \& \mathrm{C}$.

Since, $\mathrm{A}(V) \subseteq \mathrm{Ð}(V)$ we have that $\mathrm{w}=\mathrm{Aw}=\mathrm{Ð}, \forall t \in V$. From (2.1.1), $\Longrightarrow$ S. $p_{\mathrm{b}}(\mathrm{A} w, \mathrm{~B} t) \leq \mathrm{k} \cdot \max \left\{\begin{array}{c}p_{\mathrm{b}}(\mathrm{C} w, \mathrm{Ð} t), P_{\mathrm{b}}(\mathrm{C} w, \mathrm{~A} w), P_{\mathrm{b}}(\mathrm{Ð} t, \mathrm{~B} t), \\ \frac{1}{2 \dot{S}}\left[P_{\mathrm{b}}(\mathrm{C} w, \mathrm{~B} t)+P_{\mathrm{b}}(\mathrm{\oplus} t, \mathrm{~A} w)\right]\end{array}\right\}$ 


$$
\begin{aligned}
& =\mathrm{k} \cdot \max \left\{\begin{array}{c}
p_{\mathrm{b}}(w, w), P_{\mathrm{b}}(w, w), P_{\mathrm{b}}(w, \mathrm{~B} t), \\
\frac{1}{2 \hat{\mathrm{S}}}\left[P_{\mathrm{b}}(w, \mathrm{~B} t)+P_{\mathrm{b}}(w, w)\right]
\end{array}\right\} \\
& =k p_{\mathrm{b}}(w, \mathrm{~B} t) \\
& \text { It apparent this } \mathrm{B} t=w=Đ t . \\
& \because(\mathrm{B}, \mathrm{Ð}) \text { is weakly compatible, so that } \mathrm{B} w=Đ w .
\end{aligned}
$$

Again (2.1.1), $\Rightarrow$

S. $p_{\mathrm{b}}(\mathrm{A} w, \mathrm{~B} w) \leq \mathrm{k} \cdot \max .\left\{\begin{array}{c}p_{\mathrm{b}}(\mathrm{C} w, \mathrm{Ð} w), P_{\mathrm{b}}(\mathrm{C} w, \mathrm{~A} w), P_{\mathrm{b}}(\mathrm{Ð} w, \mathrm{~B} w), \\ \frac{1}{2 \dot{S}}\left[P_{\mathrm{b}}(w, \mathrm{~B} w)+P_{\mathrm{b}}(\mathrm{Ð} w, \mathrm{~A} w)\right]\end{array}\right\}$

$=$ k. $\max \left\{\begin{array}{c}p_{\mathrm{b}}(w, \mathrm{~B} w), P_{\mathrm{b}}(w, w), P_{\mathrm{b}}(\mathrm{B} w, \mathrm{~B} w), \\ \frac{1}{2 \dot{S}}\left[P_{\mathrm{b}}(\mathrm{C} w, \mathrm{~B} w)+P_{\mathrm{b}}(w, \mathrm{~B} w)\right]\end{array}\right\}$

$=k p_{\mathrm{b}}(w, \mathrm{~B} w)$

It is clear that $w=\mathrm{B} w=Đ w$.

$\therefore w$ is common fixed point of $\mathrm{A}, \mathrm{B}, \mathrm{C} \& \mathrm{D}$.

Now we demonstrate that $w$ is unique common fixed point in V. Let us assume $z$ is other common fixed point of A,B,Ç \& $Đ$.

Claim $: w=z$.

From (2.1.1), $\Rightarrow$

Ś. $p_{\mathrm{b}}(w, z) \leq \dot{S} . p_{\mathrm{b}}(\mathrm{A} w, \mathrm{~B} z)$

$\leq k \cdot \max \left\{\begin{array}{c}p_{\mathrm{b}}(C \mathrm{C} w, \oplus z), P_{\mathrm{b}}(\mathrm{C} w, \mathrm{~A} w), P_{\mathrm{b}}(\bigoplus z, \mathrm{~B} z), \\ \frac{1}{2 \hat{S}}\left[P_{\mathrm{b}}(C w, \mathrm{~B} z)+P_{\mathrm{b}}(\mathrm{D} z, \mathrm{~A} w)\right]\end{array}\right\}$

$=$ k. $\max \left\{\begin{array}{c}p_{\mathrm{b}}(w, z), P_{\mathrm{b}}(w, w), P_{\mathrm{b}}(z, z), \\ \frac{1}{2 \hat{s}}\left[P_{\mathrm{b}}(w, z)+P_{\mathrm{b}}(z, w)\right]\end{array}\right\}$

$\leq k \cdot p_{\mathrm{b}}(w, z)$.

It is clear that $w=z$.

Hence $w$ is the unique common fixed point of A, B, Ç\& Đ. The next example Clear up our substantial Theorem 2.1.

Example 2.2: Authorize $w=[0,1)$ be partial b-metric space with. $P_{\mathrm{b}}: V \times V \rightarrow[0, \infty)$ realize $\mathrm{b}$ $P_{\mathrm{b}}(v, w)=[\max .\{v, w\}]^{2}, \forall v, w \in V$. Clearly $\left(V, P_{\mathrm{b}}\right)$ is partial b-metric space with $\dot{S}=2$. Realize the mapping A, B, C, $Đ: V \rightarrow V$ by
a. $\quad \mathrm{A}(v)=\frac{V^{2}}{2 \sqrt{1+V}}, \quad \mathrm{~B}(V)=\frac{V^{2}}{4 \sqrt{1+V}}$
b. $\quad C(v)=\frac{V}{2}, \quad Đ(V)=\frac{V}{2}$.

Ç\& Đ

So $\mathrm{A}, \mathrm{B}, \mathrm{C} \& \mathrm{Ð}$ content with every stipulation of theorem (2.1) \& 0 is the unique fixed point of $\mathrm{A}, \mathrm{B}$, 


\section{CONCLUSION}

In this paper, we gave a newly fixed point theorems for Partial b-metric space. We hope that our study contributes to the development of these results by other researchers.

\section{REFERENCES}

[1] I. A. Bakhtin, The contraction principle in quasi metric spaces, It. Funct. Anal., 30 (1989), 26-37.

[2] S. Czerwik, Nonlinear set-valued contraction mappings in b-metric spaces, Atti Sem. Mat. Fis. Univ. Modena. 46 (1998), 263 - 276

[3] C. Vetroa, F. Vetrob, Common fixed points of mappings satisfying implicit relations in partial metric spaces, Journal of Nonlinear Sciences and Applications, 6 (2013), 152-161.

[4] S. G. Matthews, Partal metric topology Proc. 8th Summer Conference on General Topology and Applications, Ann. N.Y. Acad. Sci., 728 (1994), 183-197.

[5] S. Shukla, Partial b-metric spaces and fixed point theorems, Mediterranean Journal of Mathematics, doi:101007/s00009013-0327-4, (2013).

[6] A. Kaewcharoen, T. Yuying, Unique common fixed point theorems on partial metric spaces, Journal of Nonlinear Sciences and Applications, 7 (2014), 90- 101.

[7] Kranti Vithal Ghag, Ketan Shah"Conceptual Sentiment Analysis Mod" International Journal of Electrical and Computer Engineering (IJECE) Vol.8 , No.4 August, 2018. , pp. 2358 2366

[8] T. Abdeljawad, J. Alzabut, A. Mukheimer, Y. Zaidan, Banach contraction principle for Cyclical mappings on partial metric spaces, Fixed Point Theory and Applications, 2012, 2012:154, 7 pp.

[9] Abhishek Sharma, Tarun Gulat Change Detection from Remotely Sensed Images Based on Stationary Wavelet Transform International Journal of Electrical and Computer Engineering (IJECE), Vol.7, No.6, December2017, pp. 3395 3401

[10] H. Nashinea, M. Imdadb, M. Hasanc, Common fixed point theorems under rational contractions in complex valued metric spaces, Journal of Nonlinear Sciences and Applications, 7 (2014), 42-50.

[11] W. Shatanawia, H. Nashineb, A generalization of Banach's contraction principle for nonlinear contraction in a partial metric space, Journal of Nonlinear Sciences and Applications, 5 (2012), 37-43.

[12] H. Aydi, Some fixed point results in ordered partial metric spaces, Journal of Nonlinear Sciences and Applications, 3 (2011), 210 - 217.

[13] H. Aydi, M. Bota, E. Karapinar, S. Mitrovic, A fixed point theorem for set valued quasi-contractions in b-metric spaces, Fixed Point Theory and its Applications, 2012, 2012:88, 8 pp.

[14] Z. Mustafa, J. R. Roshan, V. Parvaneh, Z. Kadelburg, Some common fixed point result in ordered partial b-metric spaces, Journal of Inequalities and Applications, (2013), 2013:562.

[15] K. Jha, R.P. Pant and S.L. Singh, On the existence of common fixed points for compatible mappings, Punjab Univ. J. Math., 37 (2005), $39-48$. 